\title{
Agoraphobia and Follicle-Stimulating Hormone Levels between Tamoxifen and Goserelin versus Tamoxifen Alone in Premenopausal Hormone Receptor-Positive Breast Cancer: A 12-Month Prospective Randomized Study
}

\author{
Jung-Yoon $\mathrm{Heo}^{1 *}$, Hawoo $\mathrm{Yi}^{2,3 *}$, Maurizio Fava ${ }^{4}$, David Mischoulon ${ }^{4}$, \\ Kiwon Kim ${ }^{1,5}$, Sechang Yoon ${ }^{1}$, Hong Jin Jeon ${ }^{1,4,6} \bowtie$, and Jeong Eon Lee ${ }^{2,6} \bowtie$ \\ 'Department of Psychiatry, Depression Center, Samsung Medical Center, Sungkyunkwan University School of Medicine, Seoul, Republic of Korea \\ ${ }^{2}$ Division of Breast and Endocrine Surgery, Department of Surgery, Samsung Medical Center, Sungkyunkwan University School of Medicine, Seoul, \\ Republic of Korea \\ ${ }^{3}$ Yang Hospital, Namyangju, Republic of Korea \\ ${ }^{4}$ Depression Clinical and Research Program, Massachusetts General Hospital, Harvard Medical School, Boston, USA \\ ${ }^{5}$ National Institute of Dementia, Seongnam, Republic of Korea \\ ${ }^{6}$ Department of Health Sciences \& Technology, Department of Medical Device Management and Research, and Department of Clinical Research \\ Design \& Evaluation, Samsung Advanced Institute for Health Sciences \& Technology (SAlHST), Sungkyunkwan University, Seoul, \\ Republic of Korea
}

Objective Tamoxifen is an estrogen receptor antagonist used to prevent recurrence of breast cancer, which may provoke depression and anxiety and increase follicle-stimulating hormone (FSH) to patients. We compared anxiety and depression symptoms and FSH levels who received conventional tamoxifen alone and combination treatment of goserelin, a gonadotropin-releasing hormone (GnRH) analogue, with tamoxifen.

Methods Sixty-four premenopausal women with hormone receptor-positive early-stage breast cancer were included and were assigned randomly to receive either tamoxifen and goserelin combination or tamoxifen alone for 12 months. The participants were evaluated blindly using the Hamilton Depression and Anxiety Rating Scale, the Beck Depression Rating Scale, and the Albany Panic and Phobia Questionnaire (APPQ). Blood FSH levels were assessed at baseline, 6 and 12 months.

Results A significant timexgroup difference was detected in the agoraphobia trends subscale of the APPQ and in FSH levels. The combination group showed significantly less increases in agoraphobia subscale of APPQ and greater decreases in FSH level than those in the tamoxifen-alone group from baseline to 12 months of treatment. No significant differences for age, tumor grade, body mass index, or family history were found at baseline between the two groups.

Conclusion Our results suggest that the combination treatment of tamoxifen and goserelin resulted in less agoraphobia than tamoxifen alone in premenopausal women with breast cancer, which may associated with FSH suppression of goserelin.

Psychiatry Investig 2017;14(4):491-498

Key Words Tamoxifen, Goserelin, Anxiety, Depression, Breast cancer.

\footnotetext{
Received: March 9, 2016 Revised: July 17, 2016 Accepted: August 24, 2016 Available online: May 17, 2017

$\triangle$ Correspondence: Hong Jin Jeon, MD, PhD

Department of Psychiatry, Depression Center, Samsung Medical Center, Sungkyunkwan University School of Medicine, 81 Irwon-ro, Gangnam-gu, Seoul 06351, Republic of Korea

Tel: +82-2-3410-3586, Fax: +82-2-3410-0050, E-mail: jeonhj@skku.edu

$\triangle$ Correspondence: Jeong Eon Lee, MD, PhD

Division of Breast and Endocrine Surgery, Department of Surgery, Samsung Medical Center, Sungkyunkwan University School of Medicine, 81 Irwon-ro, Gangnamgu, Seoul 06351, Republic of Korea

Tel: +82-2-3410-3479, Fax: +82-2-3410-6982, E-mail: jeongeon.lee@samsung.com

*Jung-Yoon Heo and Hawoo Yi equally contributed this work.

(a) This is an Open Access article distributed under the terms of the Creative Commons Attribution Non-Commercial License (http://creativecommons.org/licenses/by-nc/4.0) which permits unrestricted non-commercial use, distribution, and reproduction in any medium, provided the original work is properly cited.
} 


\section{INTRODUCTION}

Breast cancer is a sex hormone-dependent disease. Approximately $60 \%$ of breast cancers in premenopausal women are hormone receptor positive (HR+). Adjuvant endocrine therapy reduces tumor recurrence and mortality in premenopausal women with estrogen receptor-positive, progesterone receptor-positive, or both. ${ }^{1}$ Recently, diverse types of approach for endocrine therapy has developed and been tested under trials.

Tamoxifen is a selective modulator of estrogen and has been the standard care for premenopausal women with $\mathrm{HR}+$ breast cancer for many ayears. ${ }^{2-4}$ The anti-cancer effect originates from binding estrogen receptors on tumors. This binding produces a nuclear complex that decreases DNA synthesis and inhibits the effects of estrogen. Tamoxifen increases plasma estradiol concentrations by interfering with the normal negative pituitary feedback mechanisms, ${ }^{5,6}$ and the resulting follicle-stimulating hormone (FSH) rise drives ovarian steroidogenesis and increased incidence of ovarian cysts. ${ }^{7}$

Goserelin is a gonadotropin-releasing hormone $(\mathrm{GnRH})$ analogue acts on the pituitary gland in the brain. ${ }^{8}$ Administration of goserelin with chemotherapy appeared to protect against ovarian failure, reducing the risk of early menopause and improving prospects for fertility. ${ }^{9}$ Initially, goserelin causes an increase in the amount of FSH and LH released from the pituitary gland, but chronic administration act by downregulating pituitary GnRH receptors, thereby suppressing the release of luteinising hormone (LH) and FSH. ${ }^{10}$ Goserelin reduces plasma/serum FSH and circulating sex hormone levels in premenopausal women to postmenopausal levels. ${ }^{11,12}$

The treatment effect of tamoxifen plus goserelin for breast cancer has been controversial. However, the recent study results of the Suppression of Ovarian Function Trial (SOFT) and the Tamoxifen and Exemestane Trial (TEXT) showed that ovarian function suppression with goserelin improved the survivals in premenopausal breast cancer patients after adjuvant chemotherapy. ${ }^{13,14}$

One of the major side effects of adjuvant chemotherapy in young patients with breast cancer is treatment-associated premature menopause. ${ }^{15}$ Many previous studies have reported that changes of hormone levels are closely related to depression and anxiety disorders. ${ }^{16-18}$ Compared to normal menopause, treatment-associated menopause is more abrupt in onset and more persistent. Treatment-associated menopause has been linked to poor health perception, leading to increased emotional stress and anxiety, particularly in young patients with breast cancer. ${ }^{19}$ Even in a general population of women without lifetime history of psychiatric diseases, women who enter the menopausal transition earlier have a significant risk for first onset of depression. ${ }^{20}$ However the adverse effects of goserelin have not been studied well in young patients with breast cancer.

The aim of this study was to compare anxiety and depression symptoms of premenopausal women with HR+ early-stage breast cancer who received either a combination of tamoxifen plus goserelin or tamoxifen therapy alone, using psychological measures, and follicle-stimulating hormone (FSH) levels, in a 12-month prospective randomized trial.

\section{METHODS}

\section{Subjects}

A total of 64 patients with breast cancer were recruited from the outpatient clinic of the Breast and Endocrine Surgery Center of Samsung Medical Center between January 2011 and December 2014. Patients were eligible to participate in the study after meeting the following inclusion criteria: age $<50$ years and premenopausal women with histologically-proven, $\mathrm{HR}+$ (defined as $\mathrm{ER} \geq 10 \%$ and/or PgR $\geq 10 \%$ ) early invasive breast cancer.

The patient exclusion criteria were: age $>50$ years, natural menopause, GnRH level $\geq 40 \mathrm{pg} / \mathrm{mL}$, pregnancy or lactation, and uncontrolled heart failure or coronary heart disease in the previous 6 months. Those with a psychotic disorder (e.g., schizophrenia or delusional disorder), bipolar affective disorder, or neurological illness, including significant cognitive impairment or Parkinson's disease, mental retardation, a significant medical condition, epilepsy, history of alcohol or drug dependence, personality disorder, or brain damage were excluded.

\section{Study procedures}

Eligible patients were randomized using a 1:1 allocation to one of the two treatment groups from the date of randomization. One group of patients received a combined treatment of tamoxifen and goserelin $(n=32)$, and the second group received tamoxifen alone $(\mathrm{n}=32)$ for 12 months. All patients attended three visits at baseline and at 6 and 12 months after treatment was initiated. Patients who were unable to tolerate the study medications per protocol were withdrawn from the study. This study protocol was approved by the ethics review board of Samsung Medical Center, Seoul, Korea. Signed informed consent was obtained from all participants.

\section{Baseline psychological evaluation}

Psychiatrists with more than 3 years of clinical experience evaluated the participants' psychiatric and medical histories and confirmed their eligibility. A trained psychologists blinded to the psychiatrists' assessment evaluated the participants' 
psychiatric diagnoses and current mood states using the Korean version of the Mini-International Neuropsychiatric Interview (MINI). ${ }^{21}$ The psychiatric evaluations were administered by a single trained rater at baseline and at 6 and 12 months.

\section{Psychological measurements}

The primary outcome measures were the Hamilton Depression Rating Scale (HAM-D) ${ }^{22}$ and the Hamilton Anxiety Rating Scale (HAM-A) ${ }^{23}$ scores of the participants. The secondary outcome measures were changes in the Beck Depression Inventory (BDI) score. ${ }^{24}$ The Albany Panic and Phobia Questionnaire (APPQ), ${ }^{25}$ and the Anxiety Sensitivity IndexRevised (ASI-R) ${ }^{26}$ scores were obtained and FSH levels were measured at each visit. In addition, Mood Disorder Questionnaire (MDQ) ${ }^{27}$ and the Hypomania Checklist (HCL-32) were employed to measure the severity of previous hypomanic episodes. $^{28}$

The current anxiety sensitivity level of the participants was assessed using the ASI-R. The Korean version of the ASI-R has demonstrated a high internal consistency coefficient ( $\alpha=0.92)$ and a good test-retest reliability $(r=0.82) .{ }^{29}$ The APPQ is a 27-item self-report questionnaire designed to measure fear of agoraphobic situations (Agoraphobia, 9 items), fear of social situations (Social phobia, 10 items), and fear of activities that produce somatic sensations (interoceptive fear, 8 items). The Korean version of the APPQ has excellent psychometric properties, which are similar to the original APPQ. ${ }^{30}$ The BDI is a well-performing, 21-item, self-report questionnaire designed to assess and evaluate the frequency of depressive symptoms over a 1-week period. We administered the Korean version of the BDI, ${ }^{31}$ which has demonstrated good psychometric properties.

\section{FSH levels}

Blood (10 mL) was taken from the cubital vein of each participant into 1) heparinized vacuum containers (plasma), 2) additive-free vacuum containers (serum), and 3) EDTA vacuum containers (for platelet count). FSH was measured using a chemiluminescence immunoassay and an ADVIA Centaur automated analyzer (Siemens Healthcare Diagnostics, New York, NY, USA). The assay detection limit was $0.3 \mathrm{IU} / \mathrm{L}$.

\section{Statistical analysis}

The chi-square or Fisher's exact test were used to compare sociodemographic and clinical categorical data between the two groups, and the t-test was used for normally distributed continuous variables. The primary efficacy measures were changes in the HAM-D and HAM-A scores and from baseline to 12 months. The Linear Mixed Model was applied to repeated measurements (after natural log transformation due to non-normality in some cases). Blood FSH levels had skewed distributions and were transformed using the natural log. The Generalized Linear Mixed model was applied to repeated ordinal outcomes. Time, group and timexgroup were considered fixed effects and subject was considered a random effect.

We performed simple and multiple linear regression analysis for univariable analysis and multivariable analysis, respectively. Variables with a p-value of less than 0.2 in the univariable analysis were included in the multivariable analysis.

Two-tailed $\mathrm{p}$-values $<0.05$ were considered significant and were corrected using Bonferroni's method for multiple testing. All statistical analyses were performed using SAS ver. 9.4 software (SAS Institute, Cary, NC, USA).

\section{RESULTS}

The demographic and clinical profiles of the patients are shown in Table 1. No differences in the demographic or clinical variables were detected at baseline between the two treatment groups. The mean age of the combined treatment group was $44.75 \pm 3.26$ years and that in the tamoxifen-alone group was $44.97 \pm 3.26$ years.

Table 2 presents the baseline psychological characteristics. Mean baseline HAM-D scores were $5.72 \pm 3.53$ vs. $6.06 \pm 3.91$ in the combined treatment and tamoxifen-alone groups, respectively. No differences were observed in HAM-D, HAMA, MDQ, HCL-32, ASI and APPQ scores at baseline (Table 2). No differences in baseline FSH levels were detected between the treatment groups (Table 1 and 2).

Table 3 shows the psychological characteristics of the patients in the two groups. A significant timexgroup difference was detected in the agoraphobia trends subscale of the APPQ and in FSH levels (Figure 1) between the two groups after 12 months of treatment. The tamoxifen and goserelin group showed significantly less of an increase in APPQ agoraphobia subscale score but a greater decrease in FSH level than those in the tamoxifen-alone group from baseline to 12 months of treatment. No significant time, group, or timexgroup differences were observed in the HAM-D and BDI score between the two groups (Table 3 and Figure 1).

A time-dependent ascending trend was detected in the total HAM-A score ( $\beta$ coefficient $=1.90, \mathrm{p}=0.01$ ) (Table 3 and Figure 1$)$, the HAM-D genital symptom subscale score ( $\beta$ coefficient=4.07, $\mathrm{p}<0.001)$, and the HAM-A intellectual $(\mathrm{p}<0.001)$, somatosensory $(\mathrm{p}<0.001)$, and autonomic symptom $(\mathrm{p}=0.01)$ subscales. The total HAM-D scores of all patients after 12 months of treatment were $\leq 7$ points (no depression). ${ }^{32}$ A total of $41.9 \%$ of the combined treatment group and $44.8 \%$ of the tamoxifen-alone group showed moderate to severe anxiety. 


\section{DISCUSSION}

This is the first study to compare anxiety and depressive symptoms and FSH levels between premenopausal women with breast cancer who received either a combined tamoxifen and goserelin treatment or a tamoxifen-alone treatment. The tamoxifen and goserelin group showed significantly less of an increase in agoraphobia symptoms but a greater decrease in FSH level than those in the tamoxifen-alone group from baseline to 12 months of treatment, whereas no differences were observed in depressive symptoms between the groups.

It is a unique finding of this study that agoraphobia symp-

Table 1. Baseline demographic and clinical characteristics of the study subjects

\begin{tabular}{|c|c|c|c|c|c|c|}
\hline \multirow{2}{*}{ Characteristics } & \multicolumn{2}{|c|}{ Tamoxifen+Goserelin group $(\mathrm{N}=32)$} & \multicolumn{2}{|c|}{ Tamoxifen group $(\mathrm{N}=32)$} & \multicolumn{2}{|c|}{ Statistics } \\
\hline & Mean & SD & Mean & SD & $\mathrm{t}$ & $\mathrm{p}$ \\
\hline Age (years) & 44.75 & 3.26 & 44.97 & 3.26 & -0.27 & 0.38 \\
\hline 35-39 yr, No. (\%) & $1(3.13)$ & & $3(9.38)$ & & & \\
\hline 40-49 yr, No. (\%) & $31(96.87)$ & & $29(90.62)$ & & & \\
\hline BMI & 22.48 & 2.00 & 23.05 & 1.95 & -1.44 & 0.26 \\
\hline Tumor size $(\mathrm{cm})$ & 1.22 & 0.39 & 0.89 & 0.51 & 2.91 & 0.01 \\
\hline \multirow[t]{2}{*}{$\mathrm{FSH}(\mathrm{mIU} / \mathrm{mL})$} & 9.45 & 8.90 & 8.81 & 7.56 & 0.32 & 0.75 \\
\hline & No. & $\%$ & No. & $\%$ & $\chi^{2}$ & $\mathrm{p}$ \\
\hline $\mathrm{FHx}(+)$ & 7 & 21.9 & 5 & 15.6 & 0.41 & 0.52 \\
\hline FHx (-) & 25 & 78.1 & 27 & 84.4 & 1.02 & 0.31 \\
\hline \multicolumn{7}{|l|}{ Nuclear grade } \\
\hline Low & 12 & 37.5 & 16 & 50.0 & 1.02 & 0.31 \\
\hline Intermediate & 20 & 62.5 & 16 & 50.0 & & \\
\hline \multicolumn{7}{|l|}{ Histological grade } \\
\hline Well & 22 & 68.8 & 26 & 81.3 & 1.33 & 0.25 \\
\hline Noderated & 10 & 31.3 & 6 & 18.8 & & \\
\hline \multicolumn{7}{|l|}{ C-erbb-2 IHC } \\
\hline Negative & 13 & 40.6 & 12 & 37.5 & 2.07 & 0.36 \\
\hline+ & 19 & 59.4 & 18 & 56.3 & & \\
\hline++ & 0 & 0 & 2 & 6.3 & & \\
\hline \multicolumn{7}{|l|}{$\mathrm{Ki}-67$} \\
\hline$\leq 14 \%$ & 19 & 59.4 & 25 & 78.1 & 2.62 & 0.11 \\
\hline$>14 \%$ & 13 & 40.6 & 7 & 21.9 & & \\
\hline
\end{tabular}

BMI: body mass index, FSH: follicle-stimulating hormone, FHx: family history, C-erbb-2 IHC: C-erbb-2 (Her-2/neu) Immunohistochemistry, Ki-67: Ki-67 protein

Table 2. Baseline psychological characteristics of the participants

\begin{tabular}{|c|c|c|c|c|c|c|}
\hline \multirow{2}{*}{ Characteristics } & \multicolumn{2}{|c|}{ Tamoxifen+Goserelin group $(\mathrm{N}=32)$} & \multicolumn{2}{|c|}{ Tamoxifen group $(\mathrm{N}=32)$} & \multicolumn{2}{|c|}{ Statistics } \\
\hline & Mean & $\mathrm{SD}$ & Mean & $\mathrm{SD}$ & $\mathrm{t}$ & $\mathrm{p}$ \\
\hline HAM-D & 5.72 & 3.35 & 6.06 & 3.91 & -0.38 & 0.71 \\
\hline HAM-A & 7.44 & 4.15 & 7.44 & 4.39 & 0 & 1 \\
\hline MDQ & 3.41 & 3.06 & 3.91 & 3.88 & -0.57 & 0.57 \\
\hline HCL32 & 5.84 & 5.47 & 6.41 & 5.747 & -0.40 & 0.69 \\
\hline ASI & 17.46 & 26.18 & 18.48 & 20.44 & -1.60 & 0.11 \\
\hline APPQ & 35.29 & 31.66 & 46.14 & 32.14 & -1.22 & 0.23 \\
\hline BDI & 8.03 & 6.12 & 7.97 & 5.37 & 0.43 & 0.97 \\
\hline
\end{tabular}

HAM-D: Hamilton Depression Rating Scale, HAM-A: Hamilton Anxiety Rating Scale, MDQ: Mood Disorder Questionnaire, HCL32: Hypomania Symptom Checklist 32, ASI: Anxiety Sensitivity Index, APPQ: Albany Panic and Phobia Questionnaire, STAI: State-Trait Anxiety Inventory 
Table 3. Psychological characteristics of the patients with breast cancer assigned randomly to 12 months of treatment with tamoxifen and goserelin or tamoxifen alone

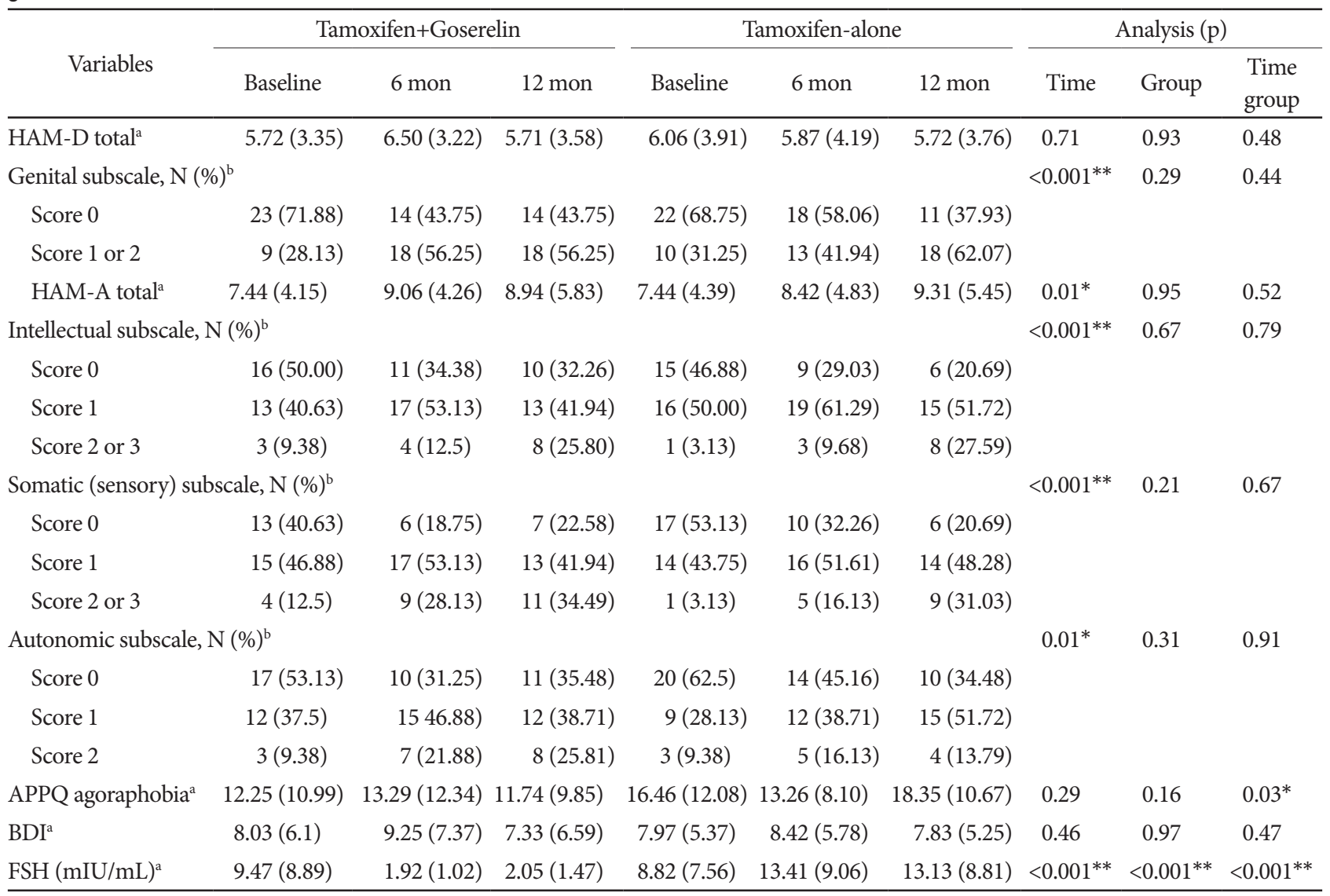

${ }^{a}$ Linear Mixed Model was applied to repeated measurements. In some cases, natural logarithm transformation was done due to non-normality. Time, group and timexgroup were considered fixed effects and subject was considered a random effect, ${ }^{b}$ Generalized Estimated Equation Model was applied to repeated ordinal outcomes. ${ }^{*} \mathrm{p}<0.05,{ }^{*} \mathrm{p}<0.01$. HAM-D: Hamilton Depression Rating Scale, HAM-A: Hamilton Anxiety Rating Scale, ASI: Anxiety Sensitivity Index, APPQ: Albany Panic and Phobia Questionnaire, BDI: Beck Depression Inventory, Genital subscale: loss of libido, impaired sexual performance, menstrual disturbances; Intellectual subscale: difficulty concentrating, poor memory; Somatic (sensory) subscale: tinnitus, blurring of vision, hot and cold flushes, feeling of weakness, pricking sensation; Autonomic subscale: dry mouth, flushing, pallor, tendency to sweat, giddiness, tension

toms are found in premenopausal women with breast cancer during anti-cancer treatment with tamoxifen. Agoraphobia is a type of anxiety symptoms which characterized by anxiety in situations where the sufferer perceives the environment to be dangerous, uncomfortable, or unsafe, which can include wide-open spaces, uncontrollable social situations, unfamiliar places, shopping malls, airports, and bridges. Although agoraphobia became independent of panic disorder in the Fifth Edition of the Diagnostic and Statistical Manual (DSM-5), agoraphobia commonly occurs in patients with panic disorder. ${ }^{33}$ Although previous preclinical studies revealed that tamoxifen treatment may provoke anxiety symptoms due to antagonize the effects of ovarian hormones in preclinical studies, ${ }^{34,35}$ human studies showed that changes in anxiety, mood, and sexual functioning were not significantly associated with treatment of tamoxifen in placebo-controlled, double-blind, randomized, controlled trials that investigated the efficacy of tamoxifen in the prevention of breast cancer in women who are at high familial risk. ${ }^{36}$

This study showed that tamoxifen and goserelin group showed significantly less of an increase in agoraphobia symptoms than tamoxifen-alone group. Goserelin is a GnRH analogue that increases FSH and luteinizing hormone (LH) synthesis, leading to increase levels of circulating sex steroids. ${ }^{37}$ In this study, FSH levels were suppressed remarkably in the combined treatment group relative to those in the tamoxifenalone group. Mean FSH level in the tamoxifen-alone group peaked within the first 6 months, and then tended to stabilize at a high level. In contrast, mean FSH level in the combined treatment group decreased and remained stable.

Previous studies have revealed that anxiety is commonly found in women in perimenopause, ${ }^{38}$ in those with premenstrual dysphoric disorder, ${ }^{39}$ and in the postpartum, ${ }^{40}$ indicating fluctuations in hypothalamic-pituitary-gonadal axis ac- 


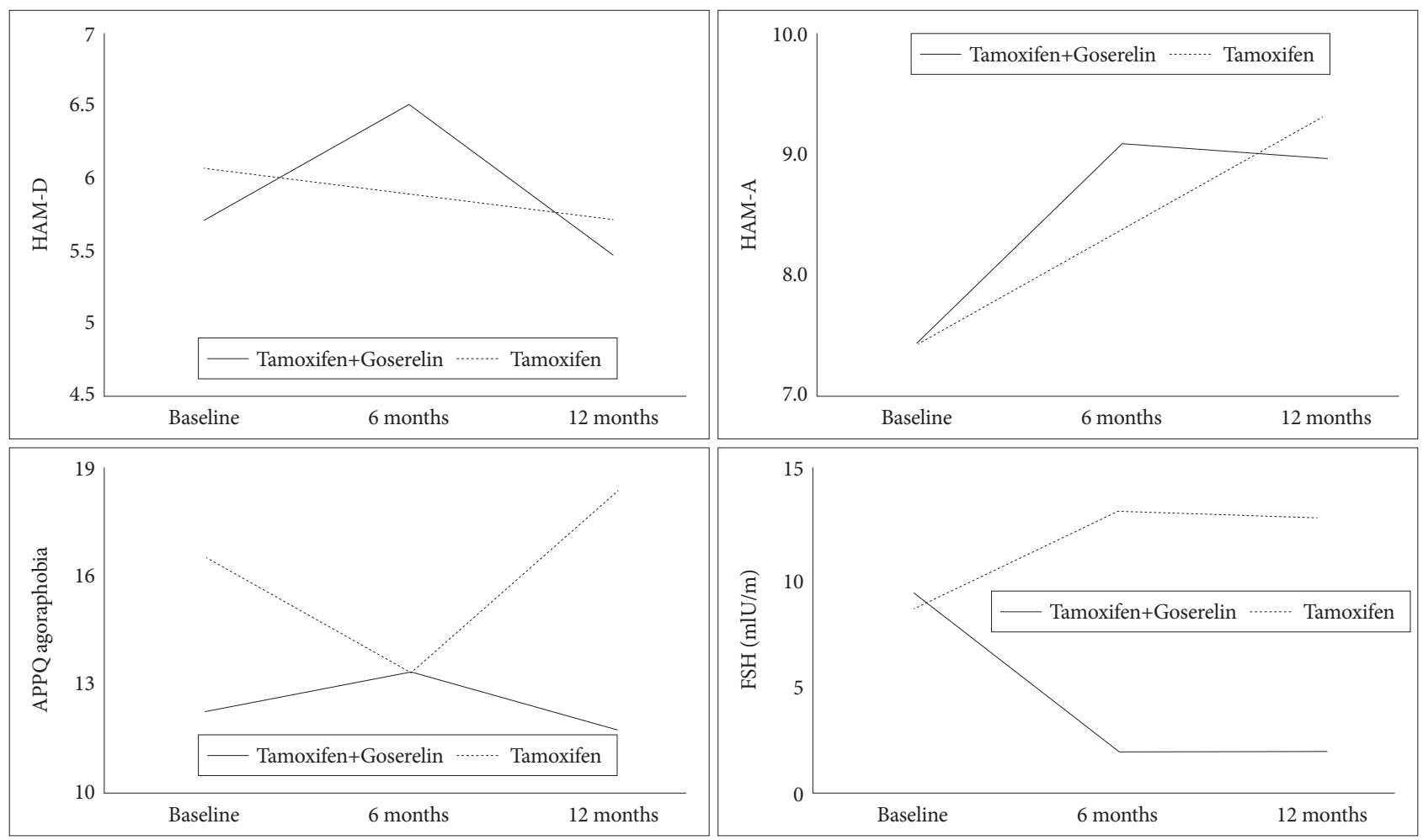

Figure 1. Linear Mixed Model Analysis and mean changes in the psychological scale scores between patients with breast cancer assigned randomly to the tamoxifen and goserelin or the tamoxifen-alone treatment. HAM-D: Hamilton Depression Rating Scale, HAM-A: Hamilton Anxiety Rating Scale, ASI: Anxiety Sensitivity Index, APPQ: Albany Panic and Phobia Questionnaire, FSH: follicle-stimulating hormone.

tivities that may result in anxiety symptoms. A previous study showed that perimenopausal fluctuation of FSH level may interact with the psychosocial environment of midlife stress to contribute to perimenopausal anxiety and depression risk through alterations in regulation of the HPA axis by $\gamma$-aminobutyric acid (GABA). ${ }^{41}$ This suggests that suppress FSH fluctuation from combination treatment of goserelin and tamoxifen can reduce anxiety symptoms among breast cancer patients, because breast cancer patients experience high stress due to cancer diagnosis, regular checks for recurrence, and body changes from breast surgery. ${ }^{42}$

Another possible effects of a decrease in estrogen levels on the serotonin system ${ }^{43,44}$ have been associated with psychiatric symptoms. Women with breast cancer are at higher risk for experiencing hot flushes, which are largely attributable to systemic cancer treatment and their effects on estrogen levels. ${ }^{45}$ Menopausal symptoms and complications, such as hot flushes, sweating, and heart palpitations, are related to anxiety in breast cancer survivors. ${ }^{46}$ Hot flush severity is also associated with higher FSH and lower estrogen levels, early menopause, beginning the late menopausal stage at a younger age, and greater anxiety. ${ }^{47}$

Our results should be considered in light of several limitations. Although the sample size was moderate, a larger sample size could offer more certain conclusions. We did not mea- sure gonadal sex steroid hormone levels, such as estrogen and progesterone; thus, we could not evaluate the direct causal relationship between anxiety and these hormone levels. The relationship between hormonal changes and mood before and after adjuvant endocrine therapy is complex and appears to be influenced by study design and multiple psychiatric and environmental factors. A future study should have a longer treatment period with more frequent follow-up visits to accurately assess changes in hormone levels and mood symptoms. More accurate clinical assessments and use of validated measures would allow menopause-related somatic complaints and psychological symptoms to be distinguished at greater levels for diagnostic and therapeutic purposes.

In conclusion, our results suggest that the combined tamoxifen and goserelin treatment for premenopausal women with breast cancer resulted in less frequent agoraphobia symptoms than those observed with tamoxifen alone. Both treatment regimens increased somatic anxiety levels. Therefore, agoraphobia and anxiety symptoms should be carefully observed in patients treated for breast cancer with anti-estrogen therapy.

\section{Acknowledgments}

This work was supported by Institute for Information \& communications Technology Promotion (IITP) grant funded by the Korea government (MSIP) (No. B0132-15-1003, the development of skin adhesive patch- 
es for the monitoring and prediction of mental disorders) and the Samsung Medical Center Clinical Research Development Program (CRDP) Grant SMO1131461 and Samsung Medical Center Grant (SMO1161491). This research was also supported by a grant of the Korea Health Technology R\&D Project through the Korea Health Industry Development Institute (KHIDI), funded by the Ministry of Health \& Welfare, Republic of Korea (HI14C3418). The authors declare no potential competing interests with respect to the research, authorship, and/or publication of this article.

\section{REFERENCES}

1. Dellapasqua S, Colleoni M, Gelber RD, Goldhirsch A. Adjuvant endocrine therapy for premenopausal women with early breast cancer. J Clin Oncol 2005;23:1736-1750.

2. Carlson RW, Allred DC, Anderson BO, Burstein HJ, Carter WB, Edge SB, et al. Breast cancer. J Nat Compr Cancer Network 2009;7:122-192.

3. Burstein HJ, Prestrud AA, Seidenfeld J, Anderson H, Buchholz TA, Davidson NE, et al. American Society of Clinical Oncology clinical practice guideline: update on adjuvant endocrine therapy for women with hormone receptor-positive breast cancer. J Clin Oncol 2010;28:37843796.

4. Goldhirsch A, Winer EP, Coates AS, Gelber RD, Piccart-Gebhart M, Thürlimann B, et al. Personalizing the treatment of women with early breast cancer: highlights of the St Gallen International Expert Consensus on the Primary Therapy of Early Breast Cancer 2013. Ann Oncol 2013;24:2206-2223.

5. Gjerde J, Geisler J, Lundgren S, Ekse D, Varhaug JE, Mellgren G, et al. Associations between tamoxifen, estrogens, and FSH serum levels during steady state tamoxifen treatment of postmenopausal women with breast cancer. BMC Cancer 2010;10:313.

6. Shapiro CL, Recht A. Side effects of adjuvant treatment of breast cancer. New Eng J Med 2001;344:1997-2008.

7. Metindir J, Aslan S, Bilir G. Ovarian cyst formation in patients using tamoxifen for breast cancer. Jpn J Clin Oncol 2005;35:607-611.

8. Nicholson RI, Walker KJ, Turkes A, Turkes AO, Dyas J, Blamey RW, et al. Therapeutic significance and the mechanism of action of the LHRH agonist ICI 118630 in breast and prostate cancer. J Steroid Biochem 1984;20:129-135.

9. Moore HC, Unger JM, Phillips KA, Boyle F, Hitre E, Porter D, et al. Goserelin for ovarian protection during breast-cancer adjuvant chemotherapy. New Eng J Med 2015;372:923-932.

10. Robertson JF, Blamey RW. The use of gonadotrophin-releasing hormone $(\mathrm{GnRH})$ agonists in early and advanced breast cancer in pre- and perimenopausal women. Eur J Cancer 2003;39:861-869.

11. Goel S, Sharma R, Hamilton A, Beith J. LHRH agonists for adjuvant therapy of early breast cancer in premenopausal women. Cochrane Database Syst Rev 2009;(4):CD004562.

12. Sverrisdottir A, Johansson H, Johansson U, Bergh J, Rotstein S, Rutqvist $\mathrm{L}$, et al. Interaction between goserelin and tamoxifen in a prospective randomised clinical trial of adjuvant endocrine therapy in premenopausal breast cancer. Breast Cancer Res Treat 2011;128:755-763.

13. Francis PA, Regan MM, Fleming GF, Lang I, Ciruelos E, Bellet M, et al. Adjuvant ovarian suppression in premenopausal breast cancer. N Engl J Med 2015;372:436-446.

14. Pagani O, Regan MM, Walley BA, Fleming GF, Colleoni M, Lang I, et al. Adjuvant exemestane with ovarian suppression in premenopausal breast cancer. N Engl J Med 2014;371:107-118.

15. Minton SE, Munster PN. Chemotherapy-induced amenorrhea and fertility in women undergoing adjuvant treatment for breast cancer. Cancer Control 2002;9:466-472.

16. Oh JY, Yu BH, Heo JY, Yoo I, Song H, Jeon HJ. Plasma catecholamine levels before and after paroxetine treatment in patients with panic disorder. Psychiatry Res 2015;225:471-475.

17. Kim B, Kang ES, Fava M, Mischoulon D, Soskin D, Yu BH, et al. Follicle-stimulating hormone (FSH), current suicidal ideation and attempt in female patients with major depressive disorder. Psychiatry Res 2013; 210:951-956.

18. Baek JH, Kang ES, Fava M, Mischoulon D, Nierenberg AA, Lee D, et al. Thyroid stimulating hormone and serum, plasma, and platelet brain-derived neurotrophic factor during a 3-month follow-up in patients with major depressive disorder. J Affect Disord 2014;169:112-117.

19. Ganz PA, Greendale GA, Petersen L, Kahn B, Bower JE. Breast cancer in younger women: reproductive and late health effects of treatment. J Clin Oncol 2003;21:4184-4193.

20. Cohen LS, Soares CN, Vitonis AF, Otto MW, Harlow BL. Risk for new onset of depression during the menopausal transition: the Harvard study of moods and cycles. Arch Gen Psychiatry 2006;63:385-390.

21. van Vliet I, de Beurs E. The MINI-International Neuropsychiatric Interview. A brief structureddiagnostic psychiatric interview for DSM-IV en ICD-10 psychiatric disorders. Tijdschr Psychiatr 2007;49:393-397.

22. Hamilton M. Development of a rating scale for primary depressive illness. Br J Soc Clin Psychol 1967;6:278-296.

23. Hamilton M. The assessment of anxiety states by rating. Br J Med Psychol 1959;32:50-55.

24. Beck AT, Ward C, Mendelson M. Beck depression inventory (BDI). Arch Gen Psychiatry 1961;4:561-571.

25. Rapee RM, Craske MG, Barlow DH. Assessment instrument for panic disorder that includes fear of sensation-producing activities: the Albany Panic and Phobia Questionnaire. Anxiety 1994-1995;1:114-122.

26. Reiss S, Peterson RA, Gursky DM, McNally RJ. Anxiety sensitivity, anxiety frequency and the prediction of fearfulness. Behav Res Ther 1986;24: $1-8$.

27. Hirschfeld RM, Williams JB, Spitzer RL, Calabrese JR, Flynn L, Keck PE Jr, et al. Development and validation of a screening instrument for bipolar spectrum disorder: the Mood Disorder Questionnaire. Am J Psychiatry 2000;157:1873-1875.

28. Angst J, Adolfsson R, Benazzi F, Gamma A, Hantouche E, Meyer TD, et al. The HCL-32: towards a self-assessment tool for hypomanic symptoms in outpatients. J Affect Disord 2005;88:217-233.

29. Lim YJ, Yu BH, Kim JH. Korean Anxiety Sensitivity Index-Revised: its factor structure, reliability, and validity in clinical and nonclinical samples. Depress Anxiety 2007;24:331-341.

30. Lee EH, Kim JH, Yu BH. Reliability and validity of the self-report version of the Panic Disorder Severity Scale in Korea. Depress Anxiety 2009;26:E120-E123.

31. Lee Y, Song J. A study of the reliability and the validity of the BDI, SDS, and MMPI-D scales. Korean J Clin Psychol 1991;10:98-113.

32. Zimmerman M, Martinez JH, Young D, Chelminski I, Dalrymple K. Severity classification on the Hamilton Depression Rating Scale. J Affect Disord 2013;150:384-388.

33. Asmundson GJ, Taylor S, Smits JA. Panic disorder and agoraphobia: an overview and commentary on DSM-5 changes. Depress Anxiety 2014; 31:480-486,

34. Azizi-Malekabadi H, Pourganji M, Zabihi H, Saeedjalali M, Hosseini M. Tamoxifen antagonizes the effects of ovarian hormones to induce anxiety and depression-like behavior in rats. Arquivos de Neuro-Psiquiatria 2015;73:132-139.

35. Mook D, Felger J, Graves F, Wallen K, Wilson ME. Tamoxifen fails to affect central serotonergic tone but increases indices of anxiety in female rhesus macaques. Psychoneuroendocrinology 2005;30:273-283.

36. Fallowfield L, Fleissig A, Edwards R, West A, Powles TJ, Howell A, et al. Tamoxifen for the prevention of breast cancer: psychosocial impact on women participating in two randomized controlled trials. J Clin Oncol 2001;19:1885-1892.

37. Ahmann FR, Citrin DL, deHaan HA, Guinan P, Jordan VC, Kreis W, et al. Zoladex: a sustained-release, monthly luteinizing hormone-releasing hormone analogue for the treatment of advanced prostate cancer. J Clin Oncol 1987;5:912-917.

38. Bromberger JT, Kravitz HM, Chang Y, Randolph JF Jr, Avis NE, Gold $\mathrm{EB}$, et al. Does risk for anxiety increase during the menopausal transi- 
tion? Study of women's health across the nation. Menopause 2013;20: 488-495.

39. Ko CH, Long CY, Chen SY, Chen IJ, Huang TH, Yen JY. Depression, irritability, and anxiety in women with premenstrual dysphoric disorder. Int J Psychiatry Med 2013;46:39-55.

40. Britton JR. Maternal anxiety: course and antecedents during the early postpartum period. Depress Anxiety 2008;25:793-800.

41. Gordon JL, Girdler SS, Meltzer-Brody SE, Stika CS, Thurston RC, Clark $\mathrm{CT}$, et al. Ovarian hormone fluctuation, neurosteroids, and HPA axis dysregulation in perimenopausal depression: a novel heuristic model. Am J Psychiatry 2015;172:227-236.

42. Antoni MH, Wimberly SR, Lechner SC, Kazi A, Sifre T, Urcuyo KR, et al. Reduction of cancer-specific thought intrusions and anxiety symptoms with a stress management intervention among women undergo- ing treatment for breast cancer. Am J Psychiatry 2006;163:1791-1797.

43. Montgomery J, Brincat M, Tapp A, Appleby L, Versi E, Fenwick P, et al. Effect of oestrogen and testosterone implants on psychological disorders in the climacteric. Lancet 1987;1:297-299.

44. Halbreich U, Kahn LS. Role of estrogen in the aetiology and treatment of mood disorders. CNS drugs 2001;15:797-817.

45. Guimond AJ, Massicotte E, Savard MH, Charron-Drolet J, Ruel S, Ivers $\mathrm{H}$, et al. Is anxiety associated with hot flashes in women with breast cancer? Menopause 2015;22:864-871.

46. Bruno D, Feeney KJ. Management of postmenopausal symptoms in breast cancer survivors. Semin Oncol 2006;33:696-707.

47. Mitchell ES, Woods NF. Hot flush severity during the menopausal transition and early postmenopause: beyond hormones. Climacteric 2015; 18:536-544. 